\title{
Test yourself answer: supra-acetabular pelvis synthetic bone graft substitute (CERAMENT) with expected resorption of radiopaque graft material
}

\author{
Molly B. Carnahan ${ }^{1}$. Jeremiah R. Long ${ }^{1}$ \\ Published online: 13 April 2020 \\ (C) ISS 2020
}

Answer: Supra-acetabular pelvis synthetic bone graft substitute (CERAMENT) with expected resorption of radiopaque graft material.

There are several bone graft substitutes commercially available in the USA. Radiologists interpreting imaging studies in patients who have undergone procedures utilizing a bone graft substitute should be familiar with their expected imaging appearance. CERAMENT (BONESUPPORT AB, Sweden) is a biphasic bone graft substitute which combines resorbable calcium sulfate $(60 \%)$ to allow bone in-growth and hydroxyapatite $(40 \%)$ to promote osteoconduction, as a scaffold for bone growth $[1,2]$. Iohexol contrast material is added to CERAMENT to improve fluoroscopic visualization during graft placement. CERAMENT has been available in the USA since 2005 and has been described in a variety of orthopedic applications including the following: treatment of benign bone lesions [3], vertebral augmentation [1], osteotomies for malunion of distal radius fractures [4], acute fractures involving the tibial plateau [5], and a reversed Hill Sachs fracture [6]. Antibiotics have also been added to CERAMENT and used in joint replacement and chronic osteomyelitis [7, 8].

After placement into the bone, CERAMENT induces bone remodeling with simultaneous dissolution and bone formation starting immediately after surgery. Due to the material's microporosity, tissue fluids penetrate the implant with nutrients and growth factors which then promote osteoclasts and macrophages to enter the material and create macropores and promote osseous in-growth [5]. On immediate postoperative

The case presentation can be found at https://doi.org/10.1007/s00256020-03430-y

Molly B. Carnahan

carnahan.molly@mayo.edu

1 Department of Radiology, Mayo Clinic Arizona, 5777 E. Mayo Clinic Boulevard, Phoenix, AZ 85054, USA radiographs, CERAMENT is expected to appear hyperdense relative to native bone, filling the entire bone void cavity. As remodeling ensues, the components of the material progressively wash out and it will therefore show a progressive decrease in radiographic density until it is completely or near completely resorbed. This resorption process has been reported to occur over a variable timeframe. Iundusi et al. reported graft material resorption within 3-8 months in a series of 24 tibial plateau fractures in which CERAMENT was utilized [5]. Horstmann et al. reported near complete graft material resorption after 1 year when CERAMENT was used in the treatment of benign and borderline bone lesions [3]. Likewise Kaczmarczyk et al. reported complete graft material resorption within 12 months in 13 out of 14 benign bone tumors in which CERAMENT was utilized in management [9]. Leakage of radiographically visible CERAMENT into surrounding soft tissues is possible and has been reported to resorb within 3-6 months [3]. Bone remodeling at the graft site is not always uniform, especially in patients in whom CERAMENT with an added antibiotic agent was utilized for the management of osteomyelitis [10]. Ferguson et al. reported a mean radiographic void filling of $73.8 \%$ and a complete void filling in $24.6 \%$ of patients in a series of 138 patients with a minimum of 1 year imaging follow-up after treatment of osteomyelitis with CERAMENT G (CERAMENT augmented with gentamycin) [10].

The most common complication associated with CERAMENT is self-limited soft-tissue inflammation. Other complications include recurrence of infection and postoperative fracture. Recurrence of infection has been reported to occur in $4-4.3 \%$ of cases after CERAMENT G $[8,10]$. The fracture rate after CERAMENT G ranges from 2.5-3\% ranging from 0.5 13 months $[8,10]$. There were no specific imaging features to suggest recurrent infection, or patients who would be at increased risk of fracture. Horstmann et al. reported an 11\% (4 of 35 cases) risk of benign rumor recurrence within 16 months. However, they reported that the rate of product resorption did not influence the indication for reoperation [3]. 
CERAMENT is a bone graft substitute with a variety of clinical applications. Due to its unique composition and expected bone remodeling after implantation, the postoperative radiographic appearance of this material is variable with complete or near complete resorption of the graft anticipated generally within 1 year. Radiologists who encounter this material in their imaging practice need to be aware of its physiologic properties and expected dynamic imaging appearances.

\section{Compliance with ethical standards}

Conflict of interest The authors declare that they have no conflicts of interest.

Ethical approval All procedures performed in studies involving human participants were in accordance with the ethical standards of the institutional and/or national research committee and with the 1964 Helsinki declaration and its later amendments or comparable ethical standards.

\section{References}

1. Marcia S, Boi C, Dragani M, et al. Effectiveness of a bone substitute (CERAMENT) as an alternative to PMMA in percutaneous vertebroplasty: 1-year follow-up on clinical outcome. Eur Spine J. 2012;21(Suppl 1):S112-8.

2. Rauschmann M, Vogl T, Verheyden A, et al. Bioceramic vertebral augmentation with a calcium sulphate/hydroxyapatite composite (CERAMENT SpineSupport): in vertebral compression fractures due to osteoporosis. Eur Spine J. 2010;19(6):887-92.
3. Horstmann PF, Hettwer WH, Kaltoft NS, Petersen MM. Early clinical and radiological experience with a ceramic bone graft substitute in the treatment of benign and borderline bone lesions. Sci Rep. 2018;8(1):15384

4. Abramo A, Geijer M, Kopylov P, Tagil M. Osteotomy of distal radius fracture malunion using a fast remodeling bone substitute consisting of calcium sulphate and calcium phosphate. J Biomed Mater Res B Appl Biomater. 2010;92(1):281-6.

5. Iundusi R, Gasbarra E, D'Arienzo M, Piccioli A, Tarantino U. Augmentation of tibial plateau fractures with an injectable bone substitute: CERAMENT. Three year follow-up from a prospective study. BMC Musculoskelet Disord. 2015;16:115.

6. Bark S, Renken F, Schulz AP, Paech A, Gille J. Arthroscopicassisted treatment of a reversed hill-sachs lesion: description of a new technique using CERAMENT. Case Rep Orthop. 2015;2015: 789203.

7. Logoluso N, Drago L, Gallazzi E, George DA, Morelli I, Romano CL. Calcium-based, antibiotic-loaded bone substitute as an implant coating: a pilot clinical study. J Bone Jt Infect. 2016;1:59-64.

8. McNally MA, Ferguson JY, Lau AC, et al. Single-stage treatment of chronic osteomyelitis with a new absorbable, gentamicin-loaded, calcium sulphate/hydroxyapatite biocomposite: a prospective series of 100 cases. Bone Joint J. 2016;98-B(9):1289-96.

9. Kaczmarczyk J, Sowinski P, Goch M, Katulska K. Complete twelve month bone remodeling with a bi-phasic injectable bone substitute in benign bone tumors: a prospective pilot study. BMC Musculoskelet Disord. 2015;16:369.

10. Ferguson J, Athanasou N, Diefenbeck M, McNally M. Radiographic and histological analysis of a synthetic bone graft substitute eluting gentamicin in the treatment of chronic osteomyelitis. J Bone Jt Infect. 2019;4(2):76-84.

Publisher's note Springer Nature remains neutral with regard to jurisdictional claims in published maps and institutional affiliations. 